\title{
FAULT ISOLATION IN TECHNICAL SYSTEMS BASED ON NON-PARAMETRIC METHOD
}

\author{
Alexey Zhirabok, Vladimir Filaretov \& Alexey Shumsky
}
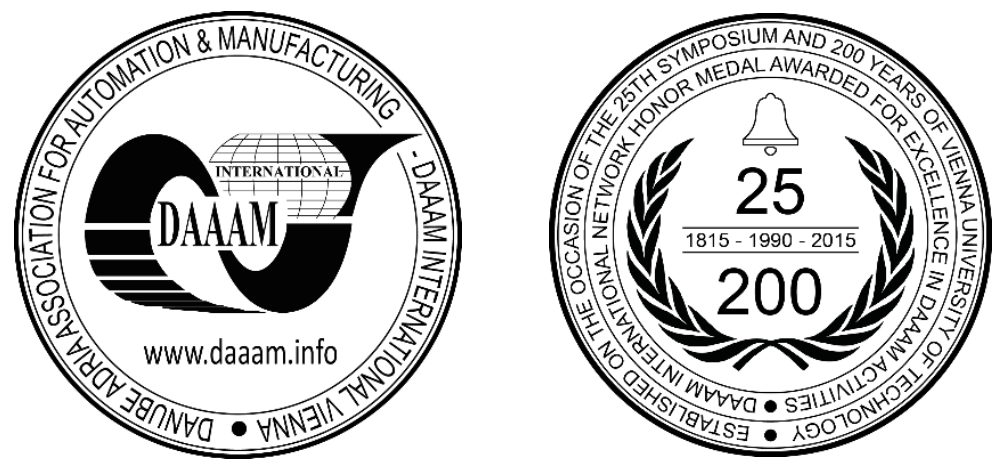

This Publication has to be referred as: Zhirabok, A[lexey]; Filaretov, V[ladimir] \& Shumsky, A[lexey] (2017). Fault Isolation in Technical Systems Based on Non-parametric Method, Proceedings of the 28th DAAAM International Symposium, pp.0150-0159, B. Katalinic (Ed.), Published by DAAAM International, ISBN 978-3-902734-11-2, ISSN 1726-9679, Vienna, Austria

DOI: $10.2507 / 28$ th.daaam.proceedings.020

\begin{abstract}
The paper is devoted to the fault isolation problem in technical systems described by nonlinear dynamic models. So-called non-parametric method is used to solve the problem. The feature of this method is that parameters of the system under consideration may be unknown. To apply this method, the system is transformed into the input-out form. The methods to obtain such a transformation and to isolate faults are suggested. They are based on so-called logic-dynamic approach. The feature of this approach is that the system transformation and fault isolation problems for nonlinear systems are solved by methods of linear algebra. The adaptive threshold is used to avoid a false alarm. The theoretical results are illustrated by practical example of diagnosis of general electric servoactuator.
\end{abstract}

Keywords: Nonlinear models; Fault isolation; Non-parametric method; Matrix of syndromes

\section{Introduction}

The problem of fault diagnosis in technical systems is very important since if a fault occurs and is not timely detected, this may results in great damages and losses. This problem was extensively investigated for the past 30 years; see, e.g., the papers [7, 8, 12, 15, 17, 19, 20], the books [2, 3, 11, 13, 14, 16, 21]. Many problems have been studied and solved: different methods of residual generation and relationships among them, robustness and adaptive threshold test, fuzzy logic, sliding mode; many types of systems have been considered: linear, bilinear, linearly-analytical, descriptor, hybrid. Many practical examples have been considered; see, for example, the books devoted to industrial and mechatronic systems $[3,14]$.

There exists a promising method of fault diagnosis in technical systems known as non-parametric, or model-free, or data-driven method (see, for example, the paper [1, 4, 6, 9, 18, 24] and the book [5]). The feature of this method is that some parameters of the system under consideration may be unknown. Besides, the method can be used for diagnosis in systems which parameters are known with limited degree of accuracy. In this paper, this method is used to solve the fault diagnosis problem in technical systems containing non-differentiable nonlinearities.

To isolate the faults, a bank of residual generators is used. Every generator from this bank is sensitive to some faults and is invariant with respect to the rest of then. There are many methods to obtain the invariance with respect to faults. They require to obtain several variants of solution and then to analyze them in order to choose the appropriate variant. In this paper, the method allowing reducing the number of variants is suggested. It is based on so-called logic-dynamic 
approach. The feature of this approach is that the problems for nonlinear systems are solved by methods of linear algebra $[22,23]$ but it does not guarantee the minimal dimension of the system designed.

Parameters of the system under diagnosis may deviate from their nominal values in some tolerance. In this case the adaptive threshold should be used to avoid a false alarm. The new method to generate such a threshold is suggested. Consider control system described by the nonlinear equations

$$
x(t+t)=F x(t)+G u(t)+C\left(\begin{array}{c}
\varphi_{1}\left(A_{1} x(t), u(t)\right) \\
\vdots \\
\varphi_{p}\left(A_{p} x(t), u(t)\right)
\end{array}\right)+\sum_{i=1}^{q} D_{i} d_{i}(t), \quad y(t)=H x(t)
$$

where $x \in R^{n}, u \in R^{m}, y \in R^{l}$ are vectors of state, control, and output, respectively; $F, G, H$, and $D_{1}, \ldots, D_{q}$ are constant matrices, $d_{1}(t), \ldots, d_{q}(t)$ are scalar functions describing the faults: if there are no faults, $d_{1}(t)=\ldots=d_{q}(t)=0$, if the $i$-th fault occurs, $d_{i}(t)$ becomes an unknown function of time; $C$ is $n \times p$ constant matrix, $A_{1}, \ldots, A_{p}$ are row matrices; $\varphi_{1}, \ldots, \varphi_{p}$ are arbitrary nonlinear functions. Notice that the linear terms in (1) are separated from the nonlinear ones, this can be done by methods described in [22, 23].

It is assumed that the matrices $F, G, C$, and $A_{1}, \ldots, A_{p}$ may contain the parameters $\gamma_{1}, \ldots, \gamma_{q}$, therewith if the parameter $\gamma_{i}$ is equal to its nominal value $\gamma_{0 i}$, then $d_{i}=0$, otherwise $d_{i} \neq 0, i=1, \ldots, q$.

\section{Problem solution}

\subsection{Preliminaries}

To implement the non-parametric methods, we need obtaining the input-output representation of system (1) or some its subsystem. Such a representation can be obtained by transformation of the state and output vectors of the initial system:

$x_{*}(t)=\Phi x(t), \quad y_{*}(t)=R y(t)$,

where $\Phi$ and $R$ are some matrices. Such a transformation yields the system described by the equations

$$
\begin{aligned}
& x_{* 1}(t+1)=x_{* 2}(t)+J_{1} y(t)+G_{* 1} u(t)+\psi_{1}\left(x_{* 2}, x_{* 3}, \ldots, y, u\right), \\
& \ldots \\
& x_{* k}(t+1)=J_{k} y(t)+G_{* k} u(t)+\psi_{k}(y, u), \\
& y_{*}(t)=x_{* 1}(t),
\end{aligned}
$$

where $x_{*_{i}}$ is the $i$-th component of the state vector $x_{*}, J_{i}$ and $G_{i}$ are the $i$-th rows of some matrices $J$ and $G, \psi_{1}, \ldots, \psi_{k}$ are nonlinear functions.

Clearly, system (2) can be transformed into the input-output form via several temporal shifts and substitutions as follows:

$$
\begin{aligned}
y_{*}(t+1)= & x_{* 2}(t)+J_{1} y(t)+G_{* 1} u(t)+\psi_{1}\left(x_{* 2}(t), x_{* 3}(t), \ldots, y(t), u(t)\right), \\
y_{*}(t+2)= & J_{1} y(t+1)+G_{* 1} u(t+1)+\psi_{1}\left(x_{*_{3}}(t)+J_{2} y(t)+G_{*_{2}} u(t)+\psi_{2}\left(x_{* 3}(t), x_{*_{4}}(t), \ldots, y(t), u(t)\right),\right. \\
& \left.x_{*_{3}}(t+1), \ldots, y(t+1), u(t+1)\right)+x_{* 3}(t)+J_{2} y(t)+G_{*_{2}} u(t)+\psi_{2}\left(x_{*_{3}}(t), x_{* 4}(t), \ldots, y(t), u(t)\right),
\end{aligned}
$$

and so forth. The last expression has the form

$$
y_{*}(t+k)=J_{1} y(t+k-1)+G_{* 1} u(t+k-1)+\ldots+J_{k} y(t)+G_{* k} u(t)+\Psi(y(t+k-1), u(t+k-1), \ldots, y(t), u(t))
$$

with some function $\Psi$. This expression can be rewritten in the form 


$$
y_{*}(t+k)=\left(\begin{array}{llllll}
J_{1} & G_{*_{1}} & \cdots & J_{k} & G_{*_{k}} & 1
\end{array}\right)\left(\begin{array}{c}
y(t+k-1) \\
u(t+k-1) \\
\cdots \\
y(t) \\
u(t) \\
\Psi(t)
\end{array}\right)
$$

Write down the expression for the variable $y_{*}$ for $T$ instants of time:

$$
Y_{T}(t)=\left(y_{*}(t+k+T-1) \quad y_{*}(t+k+T-2) \quad \cdots \quad y_{*}(t+k)\right)=\left(\begin{array}{llllll}
J_{1} & G_{*_{1}} & \cdots & J_{k} & G_{* k} & 1) V_{T}(t)
\end{array}\right.
$$

where

$$
V_{T}(t)=\left(\begin{array}{cccc}
y(t+k+T-2) & y(t+k+T-3) & & y(t+k-1) \\
u(t+k+T-2) & u(t+k+T-3) & & u(t+k-1) \\
\cdots & \ldots & \ldots & \ldots \\
y(t+T-1) & y(t+T-2) & & y(t) \\
u(t+T-1) & u(t+T-2) & & u(t) \\
\Psi(t+T-1) & \Psi(t+T-2) & & \Psi(t)
\end{array}\right) .
$$

It is assumed that the unknown parameters are in the row $\left(\begin{array}{lllllll}J_{1} & G_{*_{1}} & \cdots & J_{k} & G_{*_{k}} & 1\end{array}\right)$, the row $Y_{T}(t)$ and the matrix $V_{T}(t)$ depend on the measured inputs and outputs only. This allows to perform a diagnostic process using the row $Y_{T}(t)$ and the matrix $V_{T}(t)$, i.e. without information about values of some parameters.

\subsection{The design of linear part: general solution}

To transform system (1) into input-output representation (3) based on (2), the logic-dynamic approach will be used. According to this approach one has at first to transform the linear part of (1) described by the matrices $F$ and $G$ and then to make a transformation of the nonlinear term. Generally, the linear part of system (2) is described by the equations

$$
x_{*}(t+1)=F_{*} x_{*}(t)+G_{*} u(t)+J y(t), \quad y_{*}(t)=H_{*} x_{*}(t)
$$

with the matrices $F_{*}, G_{*}, J$, and $H$. Since $x_{*}=\Phi x$ and $y_{*}(t)=R y(t)$, it is known [7, 10, 22] that these matrices satisfy the conditions

$$
R H=H_{*} \Phi, \quad \Phi F=F_{*} \Phi+J H, \quad G_{*}=\Phi G .
$$

To construct the system in the form (2), the matrices $F_{*}$ and $H_{*}$ are found in the canonical form

$$
F_{*}=\left(\begin{array}{ccccc}
0 & 1 & 0 & \cdots & 0 \\
0 & 0 & 1 & \cdots & 0 \\
\cdots & \cdots & \cdots & \ddots & \cdots \\
0 & 0 & 0 & \cdots & 0
\end{array}\right), \quad H_{*}=\left(\begin{array}{lllll}
1 & 0 & 0 & \cdots & 0
\end{array}\right)
$$

Using matrices in (7), one can obtain from (6) equations for rows of the matrices $\Phi$ and $J$ :

$$
\Phi_{1}=R H, \quad \Phi_{i} F=\Phi_{i+1}+J_{i} H, \quad i=1, \ldots, k-1, \quad \Phi_{k} F=J_{k} H .
$$

As shown in [22], equations (8) can be transformed into the single equation $R H F^{k}=J_{1} H F^{k-1}+J_{2} H F^{k-2}+\ldots+J_{k} H$. Rewrite this equation in the form

$$
\left(\begin{array}{lllll}
R & -J_{1} & -J_{2} & \cdots & \left.-J_{k}\right) V^{(k)}=0
\end{array}\right.
$$

where 


$$
V^{(k)}=\left(\begin{array}{c}
H F^{k} \\
H F^{k-1} \\
\vdots \\
H
\end{array}\right) .
$$

\subsection{The design of linear part: fault isolation}

Consider some fault described by some matrix $D$. The transformed system is invariant with respect to this fault if $\Phi D=0$ . To take into account this condition, write down, based on (8), the expressions for three first rows of the matrix $\Phi$ :

$$
\begin{aligned}
& \Phi_{1}=R H, \quad \Phi_{2}=\Phi_{1} F-J_{1} H=R H F-J_{1} H, \\
& \Phi_{3}=\Phi_{2} F-J_{2} H=\left(R H F-J_{1} H\right) F-J_{2} H=R H F^{2}-J_{1} H F-J_{2} H .
\end{aligned}
$$

The expressions for the rest of rows can be obtained by analogy. It can be shown that the condition $\Phi D=0$ can be written in the form

$$
\left(\begin{array}{lllll}
R & -J_{1} & -J_{2} & \ldots & -J_{k}
\end{array}\right) B^{(k)}=0
$$

where

$$
B^{(k)}=\left(\begin{array}{cccc}
H D & H F D & H F^{2} D & H F^{k-1} D \\
0 & H D & H F D & H F^{k-2} D \\
0 & 0 & H D & H F^{k-3} D \\
\cdots & \cdots & \cdots & \\
0 & 0 & 0 & 0
\end{array}\right)
$$

Since the row $\left(\begin{array}{llllll}R & -J_{1} & -J_{2} & \ldots & -J_{k}\end{array}\right)$ has to satisfy the condition (9), one obtains from (9) and (11) the equation

$$
\left(\begin{array}{lllll}
R & -J_{1} & -J_{2} & \ldots & -J_{k}
\end{array}\right)\left(V^{(k)} \quad B^{(k)}\right)=0
$$

Equation (13) has a nontrivial solution if

$$
\operatorname{rank}\left(V^{(k)} B^{(k)}\right)<l(k+1)
$$

To isolate the faults, a bank of systems (5) should be used therewith each system is invariant with respect to some faults and sensitive to the rest of them. To construct the system invariant with respect to the $i$-th fault, the condition $\Phi D_{i}=0$ has to satisfy. To construct such a system, replace in (12) the matrix $D$ by $D_{i}$, find from (14) the minimal dimension $k$ and find the row $\left(\begin{array}{lllll}R & -J_{1} & -J_{2} & \ldots & -J_{k}\end{array}\right)$ satisfying (13). Then calculate the rows of the matrix $\Phi$ based on (10) and set $G_{*}:=\Phi G$.

\subsection{The design of nonlinear part}

To construct the nonlinear part of system (2), note that the general form of the nonlinear term is as follows:

$C_{*}\left(\begin{array}{c}\varphi_{1}\left(A_{*_{1}} z, u\right) \\ \vdots \\ \varphi_{p}\left(A_{*_{p}} z, u\right)\end{array}\right)=C_{*} \phi(z, u)$,

where $z=\left(\begin{array}{c}x_{*} \\ y\end{array}\right)$. It can be shown [22] that $C_{*}=\Phi C$ and

$$
A=A *\left(\begin{array}{l}
\Phi \\
H
\end{array}\right)
$$


where $A=\left(\begin{array}{c}A_{1} \\ \ldots \\ A_{p}\end{array}\right), A_{*}=\left(\begin{array}{c}A_{* 1} \\ \ldots \\ A_{* p}\end{array}\right)$.

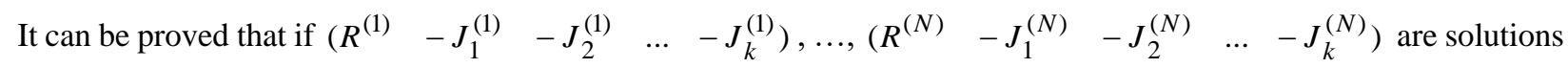
of (13) and $\Phi^{(1)}, \ldots, \Phi^{(N)}$ are the appropriate matrices, then the arbitrary linear combination of these solutions is a solution of (13) as well. This means that if $v=\left(\begin{array}{llll}v_{1} & v_{2} & \ldots & v_{N}\end{array}\right)$ is the arbitrary vector of weigh coefficients, then

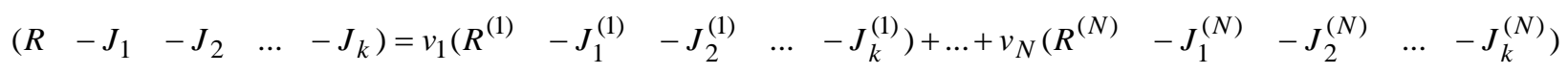

and $\Phi=v_{1} \Phi^{(1)}+\ldots+v_{N} \Phi^{(N)}$ is the matrix corresponding to $\left(\begin{array}{lllll}R & -J_{1} & -J_{2} & \ldots & -J_{k}\end{array}\right)$. Rewrite (15) as

$$
A=A_{*}^{1}\left(\begin{array}{c}
\Phi_{1} \\
\vdots \\
\Phi_{k}
\end{array}\right)+A_{*}^{2} H
$$

where $A_{*}=\left(\begin{array}{cc}A_{*}^{1} & A_{*}^{2}\end{array}\right)$. Introducing the notations

$$
\Phi_{1}^{\Sigma}=\left(\begin{array}{c}
\Phi_{1}^{(1)} \\
\vdots \\
\Phi_{1}^{(N)}
\end{array}\right), \ldots, \Phi_{k}^{\Sigma}=\left(\begin{array}{c}
\Phi_{k}^{(1)} \\
\vdots \\
\Phi_{k}^{(N)}
\end{array}\right), \quad \Phi^{\Sigma}=\left(\begin{array}{c}
\Phi_{1}^{\Sigma} \\
\vdots \\
\Phi_{k}^{\Sigma}
\end{array}\right)
$$

one may rewrite (16) in the form

$$
A=A_{*}^{1}\left(\begin{array}{c}
v \Phi_{1}^{\Sigma} \\
\vdots \\
v \Phi_{k}^{\Sigma}
\end{array}\right)+A_{*}^{2} H
$$

This equation has a solution if

$$
\operatorname{rank}\left(\begin{array}{c}
\Phi^{\Sigma} \\
H
\end{array}\right)=\operatorname{rank}\left(\begin{array}{c}
\Phi^{\Sigma} \\
H \\
A
\end{array}\right)
$$

Assuming that (18) holds, consider initially the simple case when $A$ is a row matrix. Here, (17) can be represented in the form $A=\left(\begin{array}{llll}a_{1} v & \ldots & a_{k} v\end{array}\right) \Phi^{\Sigma}+A_{*}^{2} H$, where $A_{*}^{1}=\left(\begin{array}{lll}a_{1} & \ldots & a_{k}\end{array}\right)$, or in the form

$$
A=A_{v} \Phi^{\Sigma}+A_{*}^{2} H
$$

considering $A_{v}$ as an unknown matrix. Solve this algebraic equation and find the matrices $A_{v}$ and $A_{*}^{2}$. If $A_{v}$ can be represented in the form $\left(\begin{array}{llll}a_{1} v & \ldots & a_{k} v\end{array}\right)$ for some coefficients $a_{1}, \ldots, a_{k}$ and the vector $v=\left(\begin{array}{llll}v_{1} & v_{2} & \ldots & v_{N}\end{array}\right)$, then stop, the results are the matrices $A_{*}^{1}=\left(\begin{array}{llll}a_{1} & \ldots & a_{k}\end{array}\right)$ and $A_{*}^{2}$ and the vector $v$ of weight coefficients. Then one finds the matrix $R$ and the rows of the matrices $J$ and $\Phi$ from the relations

$$
R=\sum_{i=1}^{N} v_{i} R^{(i)}, \quad J_{j}=\sum_{i=1}^{N} v_{i} J_{j}^{(i)}, \quad \Phi_{j}=\sum_{i=1}^{N} v_{i} \Phi_{j}^{(i)}, \quad j=1,2, \ldots, k,
$$

and the matrices $G_{*}=\Phi G$ and $C_{*}=\Phi C$. As a result, the nonlinear term has been constructed.

If the matrix $A$ has several rows, equation (19) is solved for all its rows with some coefficients $a_{1}, \ldots, a_{k}$ specific for each row and the vector $v$ which is the same for all rows. If the condition (18) does not hold or the matrix $A_{v}$ cannot be represented in the required form, the dimension $k$ should be increased and the described procedure is repeated. 
To check whether or not the obtained nonlinear term has the form shown in (2), consider at first the nonlinear part of the equation for $x_{*_{1}}$ formed by the first row $C_{*_{1}}$ of the matrix $C_{*}$. The product $C_{*_{1}} \phi(z, u)$ contains some rows of the form $A_{* j}$. If the first elements of these rows are equal to zero, then the product $C_{*_{1}} \phi(z, u)$ can be written as $\psi_{1}\left(x_{* 2}, x_{* 3}, \ldots, u\right)$ that corresponds to (2). Then consider the product $C_{*_{2}} \phi(z, u)$ and all rows in the form $A_{* j}$ contained in it. If the first and second elements of these rows are equal to zero, then the product $C_{* 2} \phi(z, u)$ can be written as $\psi_{2}\left(x_{* 3}, x_{* 4}, \ldots, u\right)$. The rest of the products are analyzed by analogy.

In the case when all such checks are positive, one can obtain the input-output representation of the considered system and use the non-parametric method. Otherwise, generally, the input-output representation of the considered system does not exist and one has to use other methods for diagnosis. However, in some cases, in particular, when the product $C_{*} \phi(z, u)$ contains the only nonlinearity, the possibility exists to obtain such a representation, see Section 3.

Next, the system is transformed into the input-output form (3) and (4) by performing some temporal shifts and substitutions. To generate a residual, the matrix $V_{T}(t)$ is used, see Section 2.

Note that the system invariant with respect to the $i$-th fault may be invariant with respect to some other faults. If equations in (2) depend on the parameters $\gamma_{i}, \gamma_{j}$, and $\gamma_{v}$, then the corresponding system is sensitive to the $i$-th, $j$ -th, and $v$-th faults and invariant with respect to others. To reflect these peculiarities and make a decision about faults, the matrix of syndromes $S$ is used constructed as follows. If the parameter $\gamma_{i}$ is in the $j$-th system, then $S[i, j]=1$, otherwise $S[i, j]=0$. If the $v$-th and $w$-th columns of this matrix are the same, then the $v$-th and $w$-th faults are indistinguishable in the framework of the considered approach.

\section{Decision making}

\subsection{Residual generation}

In $[4,5]$, the value of $T$ is chosen such that

$\operatorname{rank}\left(V_{T}(t)\right)=\operatorname{rank}\left(V_{T-1}(t)\right)$.

It follows from this equality that the last column of the matrix $V_{T}(t)$ is linearly dependent on the rest of columns. That is, the vector $v(T)$ exists such that $V_{T}(t) v(T)=0$ meaning that the matrix $V_{T}(t)$ has nonempty kernel. In this case, one obtains from (4) the equality $Y_{T}(t) v(T)=0$. So, the residual can be generated as

$$
r_{T}(t)=Y_{T}(t) v(T), \quad v(T) \in \operatorname{ker}\left(V_{T}(t)\right)
$$

Clearly, the rule (21) is robust in the sense that it is independent of the values of some parameters of system (1). Note that the residual should be generated on-line.

To reduce the calculation complexity, one may take $T$ such that $T>k(m+l)$; here the equality $\operatorname{rank}\left(V_{T}(t)\right)=\operatorname{rank}\left(V_{T-1}(t)\right)$ is valid. In some cases, the value of $T$ may be reduced (see example).

\subsection{Adaptive threshold}

It follows from (21) that in the unfaulty case when all parameters have their nominal values, the residual $r_{T}(t)=0$. In some cases, the parameters may vary their values slightly in some tolerances, and such variations must not be considered as faults. An adaptive threshold should be used to take into account this fact.

To design such a threshold, assume that the $i$-th parameter $\gamma_{i}$ may be presented by random variable with normal distribution having zero expectation and variance $\sigma_{i}^{2}, i=1,2, \ldots, q$. Assume also that the matrices $J$ and $G_{*}$ in (4) depend on the parameters $\gamma_{1}, \ldots, \gamma_{i}$, i.e. $J_{j}=J_{j}\left(\gamma_{1}, \ldots, \gamma_{q}\right), \quad G_{* j}=G_{* j}\left(\gamma_{1}, \ldots, \gamma_{q}\right), \quad j=1,2, \ldots, k$. For uniformity set $\Gamma=\left(\Gamma_{1}, \Gamma_{2}, \ldots, \Gamma_{2 k-1}, \Gamma_{2 k}, 1\right):=\left(J_{1}, G_{* 1}, \ldots, J_{k}, G_{*_{k}}, 1\right)$, where $\Gamma_{2 j-1}=J_{j}, \Gamma_{2 j}=G_{* j}, j=1,2, \ldots, k$.

Linearization of the last expressions yields

$$
\Delta \Gamma_{j}=\sum_{i=1}^{q} \frac{\partial \Gamma_{j}}{\partial \gamma_{i}} \Delta \gamma_{i}, \quad j=1,2, \ldots, 2 k
$$

where $\Delta \gamma_{i}=\gamma_{i}-\gamma_{0 i}, \gamma_{0 i}$ is the nominal value of the $i$-th parameter. Then 
$D\left[\Delta \Gamma_{j}\right]=\sum_{i=1}^{q} \frac{\partial \Gamma_{j}}{\partial \gamma_{i}} \sigma_{i}^{2}, \quad j=1,2, \ldots, 2 k$

Since the variable $\Delta \Gamma_{j}$ has normal distribution with zero expectation, its tolerance $\left[\Gamma_{l j}, \Gamma_{u j}\right]$ can be determined as follows:

$\Gamma_{l j}:=\Gamma_{0 j}-3\left(\sum_{i=1}^{q} \frac{\partial \Gamma_{j}}{\partial \gamma_{i}} \sigma_{i}^{2}\right)^{1 / 2}, \quad \Gamma_{u j}:=\Gamma_{0 j}+3\left(\sum_{i=1}^{q} \frac{\partial \Gamma_{j}}{\partial \gamma_{i}} \sigma_{i}^{2}\right)^{1 / 2}, \quad j=1,2, \ldots, 2 k$

where $\Gamma_{0 j}=\Gamma_{j}\left(\gamma_{01}, \ldots, \gamma_{0 q}\right)$. Set $\Gamma_{l}:=\left(\Gamma_{l, 1}, \Gamma_{l, 2}, \ldots, \Gamma_{l, 2 k-1}, \Gamma_{l, 2 k}, 1\right), \Gamma_{u}:=\left(\Gamma_{u, 1}, \Gamma_{u, 2}, \ldots, \Gamma_{u, 2 k-1}, \Gamma_{u, 2 k}, 1\right)$.

To make a decision about faults, construct the matrices $V_{T}(t)$ and $Y_{T}(t)$, find the vector $v(T)$ from $\operatorname{ker}\left(V_{T}(t)\right)$, and calculate the residual $r_{T}(t)=Y_{T}(t) v(T)$. If $r_{T}(t)=0$, one concludes that there is no fault. Otherwise calculate two values of the output $y_{*}: y_{l^{*}}(t+k+T-1)=\Gamma_{l} V_{T}^{(1)}(t)$ and $y_{u^{*}}(t+k+T-1)=\Gamma_{u} V_{T}^{(1)}(t)$, where $V_{T}^{(1)}(t)$ is the first column of the matrix $V_{T}(t)$, and check whether or not the inclusion

$y_{*}(t+k+T-1) \in\left[y_{l^{*}}(t+k+T-1), y_{u^{*}}(t+k+T-1)\right]$

is true. If it is true, one concludes that there is no fault, otherwise the fault occurred.

\section{Example}

Consider the model of the general electric servoactuator:

$$
\begin{aligned}
& \dot{x}_{1}=\frac{1}{i_{p}} x_{2}, \\
& \dot{x}_{2}=\frac{K_{M}}{J_{H}} x_{3}+\frac{M_{d}}{J_{H}} \operatorname{sign}\left(x_{2}\right), \\
& \dot{x}_{3}=-\frac{K_{\omega}}{L} x_{2}-\frac{R}{L} x_{3}+\frac{K_{U}}{L} u .
\end{aligned}
$$

Here $x_{1}$ is the output rotation angle; $x_{2}$ is the velocity at the motor output shaft; $x_{3}$ is the current through the servoactuator windings; $i_{p}$ is the reducing ratio of the reducer; $J_{H}$ is the moment of inertia of the electric servoactuator and of the rotating parts of the reducer; $K_{\omega}$ and $K_{M}$ are the respective coefficients of the counter EMF and of the torque; $M_{d}$ describes the Coulomb friction at the motor; $R$ and $L$ are the active and inductive resistances of the electric servoactuator windings, respectively; $K_{U}$ is the amplification.

Sampled-data model of the servoactuator can be obtained in the form

$$
\begin{aligned}
& x_{1}^{+}=k_{1} x_{2}+x_{1}, \\
& x_{2}^{+}=k_{2} x_{3}+k_{3} \operatorname{sign}\left(x_{2}\right)+x_{2}, \\
& x_{3}^{+}=k_{4} x_{2}+k_{5} x_{3}+k_{6} u ;
\end{aligned}
$$

for simplicity, we use the following notations: $x^{+}=x(t+1), x=x(t)$, and $u=u(t)$. We assume that $y_{1}=x_{1}, y_{2}=x_{3}$

. It is assumed also that faults are deviation of the coefficients $k_{1} \div k_{6}$ from their nominal values.

Matrices, describing system (22) and faults, are as follows:

$$
F=\left(\begin{array}{ccc}
1 & k_{1} & 0 \\
0 & 1 & k_{2} \\
0 & k_{4} & k_{5}
\end{array}\right), \quad G=\left(\begin{array}{c}
0 \\
0 \\
k_{6}
\end{array}\right), \quad H=\left(\begin{array}{ccc}
1 & 0 & 0 \\
0 & 0 & 1
\end{array}\right), \quad C=\left(\begin{array}{c}
0 \\
k_{3} \\
0
\end{array}\right), \quad A=\left(\begin{array}{lll}
0 & 1 & 0
\end{array}\right), \quad \varphi(x)=\operatorname{sign}(A x)
$$


$D_{1}=\left(\begin{array}{l}1 \\ 0 \\ 0\end{array}\right), \quad D_{2}=\left(\begin{array}{l}0 \\ 1 \\ 0\end{array}\right), \quad D_{3}=\left(\begin{array}{l}0 \\ 0 \\ 1\end{array}\right)$.

For simplicity, consider the second and the third faults; make the necessary calculations for the second fault:

$H D_{2}=\left(\begin{array}{l}0 \\ 0\end{array}\right), \quad\left(V^{(1)}, B^{(1)}\right)=\left(\begin{array}{cccc}1 & k_{1} & 0 & 0 \\ 0 & k_{4} & k_{5} & 0 \\ 1 & 0 & 0 & 0 \\ 0 & 0 & 1 & 0\end{array}\right)$

It can be shown that (13) has the solution $\left(\begin{array}{lllll}R & -J\end{array}\right)=\left(\begin{array}{lllll}k_{4} & -k_{1} & -k_{4} & k_{1} k_{5}\end{array}\right)$. Then $\Phi=\left(\begin{array}{lll}k_{4} & 0 & -k_{1}\end{array}\right), G_{*}=-k_{1} k_{6}$, $C_{*}=0$. The observer is linear and is described by the equations

$$
\begin{aligned}
& x_{*}^{+}=k_{4} y_{1}-k_{1} k_{5} y_{2}-k_{1} k_{6} u, \\
& y_{*}=x_{*_{1}}=k_{4} y_{1}-k_{1} y_{2},
\end{aligned}
$$

where $x_{*}=k_{4} x_{1}-k_{1} x_{2}$. The temporal shift yields the following result: $y_{*}(t+1)=k_{4} y_{1}(t)-k_{1} k_{5} y_{2}(t)-k_{1} k_{6} u(t)$. The expressions for $y_{*}(t+2), y_{*}(t+3)$, and $y_{*}(t+4)$ can be obtained by analogy.

Write down the relations for $y_{*}(t+1) \div y_{*}(t+4)$ in the form (4):

$\left(\begin{array}{llll}y_{*}(t+4) & y_{*}(t+3) & y_{*}(t+2) & y_{*}(t+1)\end{array}\right)=\left(\begin{array}{lll}k_{4} & -k_{1} k_{5} & -k_{1} k_{6}\end{array}\right)\left(\begin{array}{cccc}y_{1}(t+3) & y_{1}(t+2) & y_{1}(t+1) & y_{1}(t) \\ y_{2}(t+3) & y_{2}(t+2) & y_{2}(t+1) & y_{2}(t) \\ u(t+3) & u(t+2) & u(t+1) & u(t)\end{array}\right)$.

Thus, $T=4, Y_{4}^{(1)}=\left(y_{*}(t+4) \quad y_{*}(t+3) \quad y_{*}(t+2) \quad y_{*}(t+1)\right)$,

$V_{4}^{(1)}=\left(\begin{array}{cccc}y_{1}(t+3) & y_{1}(t+2) & y_{1}(t+1) & y_{1}(t) \\ y_{2}(t+3) & y_{2}(t+2) & y_{2}(t+1) & y_{2}(t) \\ u(t+3) & u(t+2) & u(t+1) & u(t)\end{array}\right), \quad r^{(1)}(4)=Y_{4}^{(1)} v(4), \quad v(4) \in \operatorname{ker}\left(V_{4}^{(1)}\right)$

Calculations for the third fault yield the following results:

$$
\begin{aligned}
H D_{1}=\left(\begin{array}{l}
0 \\
1
\end{array}\right), \quad H F D_{1}=\left(\begin{array}{c}
0 \\
k_{4}
\end{array}\right), \quad H F^{2} D_{1}=\left(\begin{array}{c}
k_{1} k_{2} \\
k_{2} k_{3}+k_{4}^{2}
\end{array}\right), \\
\left(V^{(2)}, B^{(2)}\right)=\left(\begin{array}{ccccccc}
1 & 2 k_{1} & k_{1} k_{2} & 0 & 0 & k_{1} k_{2} \\
0 & k_{3}\left(1+k_{4}\right) & k_{2} k_{3}+k_{4}^{2} & 1 & k_{4} & k_{2} k_{3}+k_{4}^{2} \\
1 & k_{1} & 0 & 0 & 0 & 0 \\
0 & k_{3} & k_{4} & 0 & 1 & k_{4} \\
1 & 0 & 0 & 0 & 0 & 0 \\
0 & 0 & 1 & 0 & 0 & 1
\end{array}\right) .
\end{aligned}
$$

Equation (13) has the solution $\left(\begin{array}{lll}R & -J_{1} & -J_{2}\end{array}\right)=\left(\begin{array}{llllll}1 & 0 & -2 & 0 & 1 & -k_{1} k_{2}\end{array}\right) ; \Phi_{1}=\left(\begin{array}{lll}1 & 0 & 0\end{array}\right), \Phi_{2}=\left(\begin{array}{lll}-1 & k_{1} & 0\end{array}\right)$, $C_{*}=C \Phi=\left(\begin{array}{c}0 \\ k_{1} k_{3}\end{array}\right)$, and $G_{*}=0$. Since we have a single solution, then $N=1$; one can check that the condition (18) holds, and $A=\left(\begin{array}{lll}0 & 1 & 0\end{array}\right)=\left(1 / k_{1}\right)\left(\Phi_{1}+\Phi_{2}\right)$. Therefore, the nonlinear term is of the form

$\phi(z, u)=\operatorname{sign}\left(\left(1 / k_{1}\right)\left(x_{*_{1}}+x_{*_{2}}\right)\right)=\operatorname{sign}\left(\left(1 / k_{1}\right)\left(y_{1}+x_{*_{2}}\right)\right)$. The observer is described by the equations

$$
\begin{aligned}
& x_{*_{1}}^{+}=x_{*_{2}}+2 y_{1} \\
& x_{*_{2}}^{+}=-y_{1}+k_{1} k_{2} y_{2}+k_{1} k_{3} \operatorname{sign}\left(\left(1 / k_{1}\right)\left(y_{1}+x_{*_{2}}\right)\right) \\
& y_{*}=x_{*_{1}}=y_{1}
\end{aligned}
$$


where $x_{*_{1}}=x_{1}, x_{*_{2}}=k_{1} x_{2}-x_{1}$. The temporal shifts yield the following results:

$y_{1}(t+1)=x_{* 2}(t)+2 y_{1}(t)$,

$y_{1}(t+2)=-y_{1}(t)+k_{1} k_{2} y_{2}(t)+2 y_{1}(t+1)+k_{1} k_{3} \operatorname{sign}\left(\left(1 / k_{1}\right)\left(y_{1}(t)+x_{*_{2}}(t)\right)\right)$,

$y_{1}(t+3)=-y_{1}(t+1)+k_{1} k_{2} y_{2}(t+1)+2 y_{1}(t+2)+k_{1} k_{3} \operatorname{sign}\left(\left(1 / k_{1}\right)\left(y_{1}(t+1)+x_{*_{2}}(t+1)\right)=\right.$

$=-y_{1}(t+1)+k_{1} k_{2} y_{2}(t+1)+2 y_{1}(t+2)+k_{1} k_{3} \operatorname{sign}\left(\left(1 / k_{1}\right)\left(y_{1}(t+1)-y_{1}(t)+k_{1} k_{2} y_{2}(t)+k_{1} k_{3} \operatorname{sign}\left(\left(1 / k_{1}\right)\left(y_{1}(t)+x_{* 2}(t)\right)\right)\right)\right.$.

It follows from the expression for $y_{1}(t+2)$ that

$-y_{1}(t)+k_{1} k_{2} y_{2}(t)+k_{1} k_{3} \operatorname{sign}\left(\left(1 / k_{1}\right)\left(y_{1}(t)+x_{*_{2}}(t)\right)\right)=y_{1}(t+2)-2 y_{1}(t+1)$.

Substituting the right hand side of this expression in the equation for $y_{1}(t+3)$, one obtains

$y_{1}(t+3)=-y_{1}(t+1)+k_{1} k_{2} y_{2}(t+1)+2 y_{1}(t+2)+k_{1} k_{3} \operatorname{sign}\left(\left(1 / k_{1}\right)\left(y_{1}(t+1)+y_{1}(t+2)-2 y_{1}(t+1)\right)\right)$.

The expressions for $y_{1}(t+4), y_{1}(t+5), y_{1}(t+6)$, and $y_{1}(t+7)$ are evident.

Assume that the coefficient $k_{1}$ is known and denote $y_{0}(t)=\operatorname{sign}\left(\left(1 / k_{1}\right)\left(y_{1}(t+1)+y_{1}(t+2)-2 y_{1}(t+1)\right)\right)$. Write down the relations for $y_{1}(t+3) \div y_{1}(t+7)$ in the form (4) with $y_{*}=y_{1}$ :

$$
\begin{aligned}
& \left(\begin{array}{lllll}
y_{1}(t+7) & y_{1}(t+6) & y_{1}(t+5) & y_{1}(t+4) & \left.y_{1}(t+3)\right)=
\end{array}\right. \\
& =\left(\begin{array}{llll}
2 & -1 & k_{1} k_{2} & k_{1} k_{3}
\end{array}\right)\left(\begin{array}{lllll}
y_{1}(t+6) & y_{1}(t+5) & y_{1}(t+4) & y_{1}(t+3) & y_{1}(t+2) \\
y_{2}(t+5) & y_{2}(t+4) & y_{2}(t+3) & y_{2}(t+2) & y_{2}(t+1) \\
y_{1}(t+5) & y_{1}(t+4) & y_{1}(t+3) & y_{1}(t+2) & y_{1}(t+1) \\
y_{0}(t+4) & y_{0}(t+3) & y_{0}(t+2) & y_{0}(t+1) & y_{0}(t)
\end{array}\right) .
\end{aligned}
$$

Thus, $T=5, Y_{5}^{(2)}=\left(\begin{array}{lllll}y_{1}(t+7) & y_{1}(t+6) & y_{1}(t+5) & y_{1}(t+4) & y_{1}(t+3)\end{array}\right)$,

$$
V_{5}^{(2)}=\left(\begin{array}{lllll}
y_{1}(t+6) & y_{1}(t+5) & y_{1}(t+4) & y_{1}(t+3) & y_{1}(t+2) \\
y_{2}(t+5) & y_{2}(t+4) & y_{2}(t+3) & y_{2}(t+2) & y_{2}(t+1) \\
y_{1}(t+5) & y_{1}(t+4) & y_{1}(t+3) & y_{1}(t+2) & y_{1}(t+1) \\
y_{0}(t+4) & y_{0}(t+3) & y_{0}(t+2) & y_{0}(t+1) & y_{0}(t)
\end{array}\right),
$$

and $r^{(2)}(5)=Y_{5}^{(2)} v(5), v(5) \in \operatorname{ker}\left(V_{5}^{(2)}\right)$.

Since the model (23) does not depend on the parameters $k_{2}$ and $k_{3}$ and (24) does not depend on $k_{4}, k_{5}$, and $k_{6}$, the matrix of syndromes takes the form

$S=\left(\begin{array}{lll}1 & 0 & 1 \\ 1 & 1 & 0\end{array}\right)$

where rows correspond to the residuals, columns to the faults.

\section{Conclusion}

The paper is devoted to the problem of fault isolation in technical systems described by nonlinear models. The nonparametric method and the logic-dynamic approach to solve this problem have been used. Based on the logic-dynamic approach, the input-output representation of the systems has been obtained. The new method to generate the adaptive threshold to avoid a false alarm has been suggested. The obtained results are illustrated by practical example of diagnosis of general electric servoactuator containing non-differentiable nonlinearity.

The future plan of researches is: (1) development of methods for residual generation based on the geometric point of view; (2) development of procedure to design the adaptive threshold to take into account the disturbances in the output vector.

\section{Acknowledgements}

This publication is supported by Russian Scientific Foundation (project No 16-19-00046). 


\section{References}

[1] Bittencourt, A.; Saarinen, K. \& Sander-Tavallaey, S. (2012). A Data-driven method for monitoring systems that operate repetitively - applications to wear monitoring in an industrial robot joint, Proc. of 8th IFAC Symp. Safeprocess'2012, pp. 198-203, Mexico.

[2] Blanke, M.; Kinnaert, M.; Lunze, J. \& Staroswiecki, M. (2006). Diagnosis and Fault-Tolerant Control, Berlin Heidelberg, Springer-Verlag.

[3] Caccavale, F. \& Villiani, L. (Eds) (2002). Fault Diagnosis and Tolerance for Mechatronic Systems, Recent Advances, Berlin Heidelberg, Springer-Verlag.

[4] Ding, S.; Wang, Y.; Yin, S.; Zhang, P.; Yang, Y. \& Ding, E. (2012). Date-driven design of fault-tolerant control systems, Proc. of 8th IFAC Symp. Safeprocess'2012, pp. 1323-1328, Mexico.

[5] Ding, S. (2014). Data-driven Design of Fault Diagnosis and Fault-tolerant Control Systems, London, SpringerVerlag.

[6] Filaretov, V.; Zhirabok, A. \& Tkachev, D. (2012). Non-parametric method for fault diagnosis in electrical circuits, Proc. of 23d Int. DAAAM Symposium, pp. 5-8, Zadar.

[7] Frank, P. (1990). Fault diagnosis in dynamic systems using analytical and knowledge-based redundancy - A survey and some new results, Automatica, Vol. 26, 1990, pp. 459-474.

[8] Gertler, J. (1993). Residual generation in model-based fault diagnosis, Control Theory and Advanced Technology, Vol. 9, 1993, 1993, pp. 259-285.

[9] Hakem, A.; Pekpe, K. \& Cocquempot, V. (2011). Sensor fault diagnosis for bilinear systems using data-based residuals, Proc. of 50th IEEE Conference on Decision and Control and European Control Conference (CDC-ECC), pp. 1576-1582, Orlando.

[10] Kwakernaak, H. \& Sivan, R. (1972). Linear optimal control systems, New York, A Division of John \& Sons, Inc.

[11] Noura, H.; Ponsart, D. \& Chamseddine, A. (2009). Fault-tolerant Control Systems. Design and Practical Applications, London, Springer-Verlag.

[12] Patton, R. (1994). Robust model-based fault diagnosis: the state of the art, Proc. of 2nd IFAC Symp. Safeprocess'1994, pp. 1-24, Espoo.

[13] Patton, R.; Frank, P. \& Clark, R. (2000). Issues of fault diagnosis for dynamic systems, London, Spring Verlag.

[14] Russell, E.; Chiang, L. \& Chiang, L. (2001). Fault Detection and Diagnosis in Industrial Systems, Berlin Heidelberg, Springer-Verlag.

[15] Samy, I.; Postlethwaite, I. \& Gu, D. (2011). Survey and application of sensor fault detection and isolation schemes, Control Engineering Practice, Vol. 19, 2011, pp. 658-674.

[16] Simani, S.; Fantuzzi, C. \& Patton, R. (2002). Model-based Fault Diagnosis in Dynamic Systems Using Identification, Berlin Heidelberg, Springer-Verlag.

[17] Shumsky, A. \& Zhirabok, A. (2006). Nonlinear diagnostic filter design: algebraic and geometric points of view, Int. Journal of Applied Mathematics and Computer Science, Vol. 16, 2006, pp. 115-127.

[18] Shumsky, A. (2009). Functional diagnosis of nonlinear time-delay dynamic systems, Automation and Remote Control, Vol. 70, 2009, pp. 172-184.

[19] Tan, C. \& Edwards, C. (2009). Robust fault reconstruction using multiple sliding mode observers in cascade: development and design, Proc. of 2009 American Control Conference, pp. 3411-3416, St. Louis.

[20] Witczak, M.; Korbicz, J. \& Jrozefowicz, R. (2013). Design of unknown input observers for non-linear stochastic systems and their application to robust fault diagnosis, Control and Cybernetics, Vol. 42, 2013, pp. 1-30.

[21] Yang, H.; Jiang, B. \& Cocquempot, V. (2010). Fault Tolerant Control Design for Hybrid Systems, In Lecture Notes in Control and Information Sciences, Berlin Heidelberg, Springer-Verlag.

[22] Zhirabok, A. \& Usoltsev, S. (2000). Linear diagnosis methods for nonlinear systems, Automation and Remote Control, Vol. 61, 2000, pp. 201-1209.

[23] Zhirabok, A.; Kucher, D. \& Filaretov, V. (2010). Achieving robustness at diagnosis of nonlinear systems, Automation and Remote Control, Vol. 71, 2010, pp. 142-155.

[24] Zhirabok, A. \& Pavlov, S. (2015). Data-driven method of fault detection in technical systems, Procedia Engineering, Vol. 100, 2015, pp. 242-248. 